\title{
OBSERVACIONES EN TORNO AL SENTIDO ÉTICO \\ DEL TRACTATUS LOGICO-PHILOSOPHICUS \\ Y A LA IMPORTANCIA DE LO INEFABLE*
}

\author{
David Pérez Chico \\ Universidad de Zaragoza \\ davidpch@unizar.es
}

\section{Resumen}

En los cien años que han transcurrido desde su publicación, el Tractatus Logico-Philosophicus de Wittgenstein se ha convertido en un clásico contemporáneo con la peculiaridad de que está rodeado por un aura de misterio: ¿cuál es realmente el objetivo de Wittgenstein al escribir el Tractatus?, ¿`ómo debemos entender las dos últimas proposiciones del Tractatus que nos conminan a desprendernos del libro mismo y a guardar silencio?, ¿puede el Tractatus ser otra cosa distinta a un tratado positivista y antimetafísico?, etc. Aquí sostendremos que la respuesta a estas preguntas debe reparar en cuál puede ser el sentido ético del Tractatus y, estrechamente relacionado con eso, cuál es su importancia y la de lo inefable en general. Palabras clave: Wittgenstein, Tractatus Logico-Philosophicus, ética, importancia de lo inefable, lo místico.

\author{
OBSERVATIONS ON THE ETHICAL MEANING \\ OF THE TRACTATUS LOGICO-PHILOSOPHICUS \\ AND THE IMPORTANCE OF THE INEFFABLE
}

\section{Abstract}

In the hundred years since its publication, Wittgenstein's Tractatus Logico-Philosophicus has become a contemporary classic with the peculiarity that it is surrounded by an aura of mystery: what is really Wittgenstein's aim in writing the Tractatus, how are we to understand the last two propositions of the Tractatus that enjoin us to detach ourselves from the book itself and keep silent, can the Tractatus be anything other than a positivist and anti-metaphysical treatise, and so on? Here we will argue that the answer to these questions must consider what the ethical meaning of the Tractatus might be and, closely related to that, what its importance is and that of the ineffable in general.

Keywords: Wittgenstein, Tractatus Logico-Philosophicus, ethics, the importance of the unsayable, the mystical. 
¡Muestra, no cuentes! ¿No es lo que preferís los guionistas?

(Westworld, ep. 1.8)

El Tractatus Logico-Philosophicus de Wittgenstein es un clásico contemporáneo con la peculiaridad de que está rodeado por un aura de misterio: ¿cuál es realmente el objetivo de Wittgenstein al escribir el Tractatus?, ¿`cómo debemos entender las dos últimas proposiciones del Tractatus que nos conminan a desprendernos del libro mismo y a guardar silencio?, ¿puede el Tractatus ser otra cosa distinta a un tratado positivista y antimetafísico?, etc. Como todos los clásicos, el Tractatus sigue despertando el interés de miles de lectoras y lectores y les sigue diciendo cosas, si bien cómo las dice sigue siendo un problema en sí mismo para cualquiera que trate de comprender el libro. Además, no se caracteriza Wittgenstein por hacer concesiones a sus lectores. Si acaso se limita a anunciar, al comienzo mismo del "Prólogo», que obtendrán mayor provecho de las enseñanzas del libro aquellas personas que hayan pensado por sí mismas las cuestiones de las que trata:

Posiblemente solo entienda este libro quien ya haya pensado alguna vez por sí mismo los pensamientos que en él se expresan o pensamientos parecidos. No es, pues, un manual. Su objetivo quedaría alcanzado si procurara deleite a quien, comprendiéndolo, lo leyera ${ }^{1}$.

De alguna manera, entonces, el Tractatus anuncia las condiciones de su comprensión. El libro exige a sus lectores traer los deberes hechos, por así decirlo. Son ellos los que tienen que poner de su parte, por ejemplo, reconociendo lo que el Tractatus muestra, siendo el único asidero a su disposición la de su propio interés.

Aunque he empezado admitiendo que cuál pueda ser el objetivo del Tractatus es una cuestión abierta al debate y la polémica, no es menos cierto que contamos con algunos testimonios de puño y letra de Wittgenstein que ofrecen pistas sólidas para formarnos una idea cabal de cuál puede ser ese objetivo, solo que ninguno de

* Aunque con modificaciones importantes de enfoque y de contenido, este trabajo es una versión de otro trabajo anterior titulado «O sons do silêncio. Observaçoes sobre a importantância do inefável no Tractatus Logico-Philosophicus» (Dall'Agnol, Darlei y Peruzzo Júnior, Leo (orgs.), TLP 100: Revisitando a obra de Wittgesntein, Curitiba: Florianopolis: PUCPress, EDUFSC, 2021). Agradezco a los organizadores del libro que contiene mi trabajo, Darlei Dall'Agnol y Leo Peruzzo, su generosidad a la hora de permitirme publicar esta versión del mismo.

** Este trabajo forma parte de los proyectos «Perspectivas personales. Conceptos y aplicaciones» (RTI2018-098254-B-100. Ministerio de Ciencia, Innovación y Universidades. Programa Estatal de I+D+i Orientada a los Retos de la Sociedad. Gobierno de Espańa) y «Comprensión intercultural, pertenencia y valor: aproximaciones wittgensteinianas» (PGC2018-093982-B-100. Ministerio de Economía y Competencia. Gobierno de España).

1 Wittgenstein, L. Tractatus Logico-Philosophicus. Traducción de Jacobo Muñoz e Isidoro Reguera, Madrid: Alianza, 1999, p. 11. (Tractatus a partir de aquí). 
esos testimonios zanja definitivamente el debate, sino que hay que procurar hacerlos compatibles con las proposiciones del Tractatus. Voy a ocuparme, tan solo, de dos de esos testimonios. En primer lugar, y nuevamente en el «Prólogo» del Tractatus, podemos leer lo siguiente:

El libro quiere, pues, trazar un límite al pensar o, más bien, no al pensar, sino a la expresión de los pensamientos: porque para trazar un límite al pensar tendríamos que poder pensar ambos lados de este límite (tendríamos, en suma, que poder pensar lo que no resulta pensable).

Así pues, el límite sólo podrá ser trazado en el lenguaje y lo que reside más allá del límite será simplemente absurdo².

Se trata, así pues, de delimitar el ámbito de lo que puede ser dicho con sentido, o del sentido a secas. Más allá de los límites del sentido quedará el sinsentido, aunque siendo estrictos, nada podemos decir sobre lo que hay exactamente más allá de esos límites porque no puede ser pensado. El Tractatus se propone lograr este objetivo explicando cómo es posible decir algo con sentido. Y lo hace por medio de lo que se conoce como la teoría figurativa del significado o simplemente teoría de la figura ${ }^{3}$.

2 Tractatus, p. 11.

3 Voy a asumir que mis potenciales lectores hacen gala de una cierta familiaridad con esta teoría y por ello me ahorro entrar en detalles en el texto principal, lo cual lo alargaría innecesariamente. No obstante, no está de más recordar sus puntos básicos. En primer lugar, recordemos que para Wittgenstein el mundo es la totalidad de los hechos o estados de cosas, no de objetos. Aquellos están formados por combinaciones de estos. El sentido de una proposición es el estado de cosas del mundo por ella representado o descrito, y decimos que una proposición con sentido es una figura (se dice que la proposición figura -representa o describe-el estado de cosas que es su sentido). Para que la proposición tenga sentido es suficiente con que el estado de cosas sea posible, es decir, no tiene que ser necesariamente el caso, aunque, si lo es, la proposición, además de tener sentido, es verdadera ( $c f r$. Tractatus 4.25). Voy a limitarme al caso de las proposiciones lingüísticas u oraciones, aunque Wittgenstein elabora en primer lugar una teoría aplicable a cualquier tipo de figura: nos hacemos figuras de los hechos (Tractatus 2.1) y una figura es un modelo de la realidad (Tractatus 2.12). Ahora bien, ¿qué es lo que hace que una proposición sea una figura? Tengamos en cuenta que, así como un estado de cosas está compuesto por objetos más simples que se combinan entre ellos de una determinada manera, dando lugar al estado de cosas en cuestión, también las proposiciones están compuestas por nombres que, por un lado, se corresponden en una relación uno a uno con los objetos del estado de cosas figurado (un nombre significa el objeto [Tractatus 3.203]), y, por otro lado, se combinan entre ellos de la misma manera en que se combinan los objetos del estado de cosas: comparten su forma. Además de los nombres que nombran directamente objetos, tenemos proposiciones elementales, que básicamente son concatenaciones de nombres (Tractatus 4.22), y la concatenación de estas da lugar a proposiciones más complejas que son función de verdad de las proposiciones elementales (Tractatus 5 ). Decimos que se produce un isomorfismo estructural entre el estado de cosas figurado (y en general el mundo) y la proposición que lo figura (el lenguaje en general). Este isomorfismo lo hace posible la lógica, es decir, las combinaciones posibles entre objetos y entre nombres obedecen a la lógica. Es por el hecho de que una proposición y el estado de cosas que constituye su sentido comparten su forma por lo que el lenguaje, en tanto que conjunto de proposiciones, no solo muestra cómo puede ser el mundo, sino que además dice cómo es el mundo si lo que representa es el caso. Los límites de lo que puede ser expresado con sentido, en definitiva, los establece la lógica. Y en tanto límite, nada 
Nos encontramos con un pasaje, por lo demás, claramente compatible con la recepción analítica del Tractatus que siempre ha considerado que se trata de uno de los hitos fundamentales del llamado giro lingüístico ${ }^{4}$. Otro aspecto que parece justificar esta recepción es la idea de que, tal y como queda dicho también en el prólogo, el libro comunica verdades: «me parece que la verdad de los pensamientos de los que se da cuenta aquí es intocable y definitiva. Soy por ello de la opinión de que, en lo esencial, he resuelto los problemas de modo indiscutible». Sin embargo, en una conocida carta que envía a su amigo y editor Ludwig Von Fricker después de que el Tractatus hubiera sido rechazado por varias editoriales, confiesa esto otro:

... la finalidad del libro es ética. Una vez quise poner en el prefacio unas palabras que ya no figuran en él, las cuales, sin embargo, se las escribo a usted ahora porque pueden darle una clave: quería escribir que mi libro constaba de dos partes: la que está escrita, y de todo lo que no he escrito. Y precisamente esa segunda parte es la más importante. Pues la ética queda delimitada desde dentro, como si dijéramos, por mi libro; y estoy convencido de que, en rigor, SÓLO puede delimitarse de este modos.

Hay en la comunidad de intérpretes del Tractatus quienes, como por ejemplo Fairhust (2021) por citar un caso reciente, mantienen que no debemos conceder a esta carta una importancia excesiva a la hora de interpretar el Tractatus ${ }^{6}$. Por mi

puede ser dicho sobre ello: «las proposiciones pueden representar toda la realidad, pero no pueden representar lo que tienen en común con la realidad para poder representarla: la forma lógica» (Tractatus 4.12). Nada puede decirse sobre cómo figura una proposición un estado de cosas el mundo.

${ }^{4}$ Michael Dummett, por ejemplo, afirma la siguiente: «Si identificamos el giro lingüístico como el punto de partida de la filosofía analítica propiamente dicha, no cabe duda de que, por mucho que Frege, Moore y Russell hayan preparado el terreno, el paso crucial lo dio Wittgenstein en el Tractatus Logico-Philosophicus de 1922" (Dummett, M. Origins of Analytical Philosophy. Londres: Duckworth, 1993, p. 122).

${ }^{5}$ La carta continúa: «En resumen, creo: Todo aquello acerca de lo cual muchos aún parlotean hoy en día lo he defendido en mi libro guardando silencio. Por tanto, si no me equivoco, el libro tendrá muchas cosas que decir que usted mismo querría decir, pero quizá no notará que se dicen» (Monk, R. Wittgenstein, el deber de un genio. Barcelona: Anagrama, 1994, p. 177).

${ }^{6}$ A Fairhust le parece que una lectura que conceda demasiado crédito a lo que Wittgenstein le dice a Von Fricker acerca de que el objetivo del Tractatus es ético «exagera la importancia de la carta» y con ello "devalúa el Tractatus en tanto que una obra completa de filosofía que se las ve con una multitud de cuestiones que exceden lo ético». Y añade: «Una breve carta escrita privadamente para Von Fricker acerca de una enmienda al prólogo del Tractatus no debería ser considerada como una fuente más respetable de evidencia textual que los contenidos reales del Tractatus. En otras palabras: las aportaciones de Wittgenstein en la carta acerca de su contribución a la ética en el Tractatus deben ser usados para complementar, no para negar ni sustituir, lo que ya se ha indicado en el Tractatus" (J. Fairhust, "The Ethical Significance of the Tractatus Logico-Philosophicus». Teorema XL/2, 2021, p. 155). Y en esto último es donde creo que Fairhust yerra en su apreciación. Al decir que el objetivo del libro es ético, Wittgenstein no está afirmando que el Tractatus sea, strictu sensu, una aportación a la ética como disciplina, sino que el resultado de comprender lo «que ya se ha indicado en el Tractatus» es, lato sensu, de naturaleza ética. Aún tenemos que ver qué quiere decir esto exactamente. Me gustaría añadir que para que el libro tenga un objetivo ético o, incluso, que el Tractatus mismo sea ético, no es 
parte creo que no es exagerado concederle una importancia decisiva, pues en ella se dan cita juntos tres aspectos que a mi modo de ver son cruciales para comprender lo que persigue conseguir Wittgesntein en el Tractatus. En primer lugar (1) que la finalidad del Tractatus es ética, si bien, en segundo lugar; (2) no hay proposiciones éticas en el libro (no dice nada explícitamente ético) porque la ética es trascendente, se sitúa más allá de lo que se puede decir (los límites de lo que puede ser dicho con sentido también sirven para delimitar "desde dentro» a lo que no puede ser dicho); $y$, en tercer lugar, (3) eso que queda más allá de los límites de sentido, aquello sobre lo que Wittgenstein no ha escrito nada (porque es inefable) es lo importante (es decir, es más importante que lo que sí dice el Tractatus).

Reparemos en que, en el momento de escribir la carta dirigida a Von Fricker, el libro ya había sido rechazado por varios editores y eran muy pocas, prácticamente ninguna, las esperanzas que tenía Wittgenstein de publicarlo; tengamos en cuenta, además, que alguno de esos editores le había sugerido la posibilidad de pagar la edición del libro, y que Wittgenstein se había negado rotundamente a tomar esta clase de atajo. Por todo ello, y por lo que conocemos de la estricta personalidad de nuestro autor, me parece que esta es, antes que cualquier otra cosa, un intento completamente honesto por parte de quien no tiene nada que perder de confiarse a un conocido, que además resulta ser editor.

No cabe duda, no obstante, de que se trata de un intento ciertamente llamativo porque, en la carta, Wittgenstein hace precisamente lo que según el Tractatus no se puede hacer: dice algo sobre lo que nada puede ser dicho, sobre aquello cuya comprensión depende completamente de que los lectores sean capaces de reconocer lo que la «armonía interna» del libro les muestra ${ }^{7}$, y por esa razón las proposiciones

necesario que contenga proposiciones explícitamente éticas, sino que puede darse el caso de que, por así decirlo, la ética permee toda la obra, que puede, entonces, seguir siendo una obra de filosofía tan completa como se quiera sin dejar de ser, en algún sentido, ética. Creo que esta es una característica no solo del Tractatus, sino de todo el corpus wittgensteiniano, que fía el avance o progreso de la filosofía a un cambio de actitud con respecto a lo que esta tiene por importante, por ejemplo.

${ }^{7}$ Como en el caso anterior de la teoría figurativa del significado, estoy dando por sentado que la distinción «decir» / «mostrar» es lo suficientemente conocida como para poder ahorrarme explicaciones (para una detallada interpretación de esta distinción puede verse, por ejemplo, CAROLIS, M. de «Saying and Showing. How can we communicate that of which we cannot speak?». En Lütterfelds, W., Majetschak, S., Raatzsch, R. y Vosseenkuhl, W. (eds.), Wittgenstein-Studien, vol. 4, n. ${ }^{\circ}$ 1, Berlin/Nueva York: De Gruyter, 2013, pp. 129-142; y también SANFÉLIX, V. «Mostrar y decir. Una diferencia estética».ethic@, v. 15, n. ${ }^{\circ} 2,2016$, pp. 328-342. No obstante, es crucial que en esto estemos en sintonía, así que además de lo que pueda decir más adelante, añadiré aquí tan solo que se trata de dos términos a los que Wittgenstein otorga un sentido técnico, de tal manera que decir algo significa que lo decimos o afirmamos con pretensión de verdad y que de alguna manera podría ser constatado objetivamente (¿se corresponde la figura con el estado de cosas figurado?); mientras que al mostrar algo no se afirma nada con respecto a cuál pueda ser su valor de verdad. Un ejemplo trivial: decir que, en la habitación, puede que, escondidas, hay "una pelota azul y otra blanca» es afirmar que tal cosa es verdad con respecto a una determinada situación en el mundo, pero también muestra, sin decirlo, que tal cosa es una posibilidad en nuestro mundo, así como que hay más de una pelota. El ejemplo más famoso dentro del corpus wittgensteiniano de lo que es exactamente 
del Tractatus serían sinsentidos y, como afirma la célebre proposición 6.54, habría que desprenderse de ellas: ${ }^{8}$

Mis proposiciones son elucidaciones de este modo: quien me entiende las reconoce al final como sinsentidos, cuando mediante ellas - a hombros de ellas- ha logrado auparse por encima de ellas. (Tiene, por así decirlo, que tirar la escalera una vez que se ha encaramado en ella).

Tiene que superar esas proposiciones; entonces verá el mundo correctamente» (Tractatus, 6.54).

Exploraré la siguiente propuesta: la visión correcta del mundo que debe proporcionarnos la comprensión del libro es una visión liberadora de los corsés epistémicos (representacionalistas, cientificistas) que limitan nuestro mundo al convertirlo

mostrar algo quizá sea el del poema de Ludwig Uhland El espino del Conde Eberhard, que su amigo Engelman envió a Wittgenstein el 9 de abril de 1917. En el poema se cuentan las tribulaciones de un caballero cruzado que después de regresar de Tierra Santa, plantó en el jardín de su hacienda el esqueje de un espino que había traído consigo. Su nueva vida ajena a las batallas del pasado es una vida contemplativa. Nuestro caballero recuerda el tiempo pasado mientras observa crecer al espino en su jardín. Pues bien, el motivo por el que Engelman se decide a enviarle el poema a Wittgenstein es porque, sin decirlo, el "poema entero ofrecía [...] la imagen de una vida» (Wittgenstein, L. y Engelman, P. Cartas, recuerdos, encuentros. Valencia: Pre-Textos, 2009, p. 137).

${ }^{8}$ Este es otro aspecto fundamental para comprender el Tractatus que también me limitaré a comentar muy rápidamente en esta nota al pie y no en el texto principal. Ya sabemos cuándo una proposición tiene sentido y en qué consiste este. Las proposiciones con sentido muestran su sentido (un estado de cosas posible) y además dicen que el mundo es así, si son verdaderas. No es difícil colegir a partir de aquí que una proposición será un sinsentido cuando no tenga sentido, es decir, cuando pretendiendo decir algo (i. e., representar un estado de cosas del mundo) no pueda en realidad decir nada (por ejemplo, porque sus constituyentes no nombran nada). En otras palabras: una proposición es un sinsentido cuando no logra ser una figura de la realidad. Pero, atención, estrictamente hablando hay dos maneras en las que una proposición puede no tener sentido: en primer lugar tenemos las proposiciones que, como las de la lógica (tautologías o contradicciones), carecen de condiciones de verdad (las tautologías porque son «incondicionalmente verdaderas", y las contradicciones porque no son verdaderas «bajo condición alguna» [Tractatus 4.461]); en este caso hablamos de proposiciones carentes de sentido (Sinnlos) que no dicen nada acerca de la realidad, no representan ningún estado de cosas. Pero no son completos sinsentidos porque «describen el armazón del mundo o, más bien, lo representan» (Tractatus 6.124). Esto es, las proposiciones lógicas muestran las propiedades del mundo y del lenguaje que lo describe. Así que según Wittgenstein se sitúan justo en el límite del sentido. Hay otro tipo de proposiciones que no tienen sentido pero que, a diferencia de las anteriores, estas sí, traspasan el límite del sentido. Al igual que las proposiciones de la lógica, tampoco estas dicen nada acerca de la realidad, pero a diferencia de ellas estas sí pretenden decir algo, por ejemplo, sobre las condiciones de posibilidad del sentido o sobre el valor (en este caso hablamos de proposiciones sinsentido: Unsinn). Estas proposiciones también muestran propiedades lógicas de nuestro mundo y del lenguaje que usamos para describirlo (el ejemplo que pone el propio Wittgenstein y que encontramos citado constantemente se vale de la diferencia entre la proposición «llueve o no llueve»-que es una proposición que al mostrar una propiedad lógica de nuestro mundo agota todas las posibilidades- y "llueve», dicho sin añadir más información que nos ayude a contextualizar lo que se pretende decir). En este último caso, aunque realmente no se dice nada, sí que se muestra una propiedad de nuestro mundo (i.e. que se trata de un mundo en el que la lluvia es una posibilidad). 
en un objeto más de conocimiento: en un algo acabado. Desprenderse de las proposiciones del propio Tractatus no es realmente un sacrificio, sino una necesidad, porque cualquiera que quiera ver el mundo correctamente tiene que desprenderse de sus convicciones o prejuicios, entre otros, epistémicos ${ }^{9}$. Frege y Russell, por lo que sabemos, o no veían la necesidad de hacerlo o no estaban preparados para hacerlo, y tampoco parece haberlo estado la recepción analítica del libro ${ }^{10}$. Esta resistencia monolítica a aceptar que pueda haber un tipo de logro sustantivo diferente a la ratificación o justificación del contenido de nuestros pensamientos y proposiciones se traduce en una interpretación ortodoxa según la cual el Tractatus condena a la metafísica a guardar el más absoluto de los silencios y a habitar las profundidades del sinsentido y la incomunicación. Mientras el acento se ponga en los aspectos positivistas y antimetafísicos del Tractatus, en su realismo descriptivista, etc., Wittgenstein se encontrará muy solo en la atalaya desde la que se ha desprendido de la escalera que le llevó hasta allí arriba ${ }^{11}$ y, por lo tanto, no tendrá a nadie con quien hablar, pero pocos silencios son tan elocuentes.

9 Algo parecido es lo que hace el propio Wittgenstein y de paso es lo que otorga a sus escritos un cierto tono melodramático, que si bien es más acusado en su obra de madurez ( $c f$. WitTGenstein, L. Investigaciones filosóficas, Barcelona: Crítica, 1988, \$118), ya está presente de manera clara en algunos pasajes del Tractatus. Esto se debe a que Wittgenstein pretender superar la filosofía tradicional u ortodoxa (sus métodos y problemas, lo que tiene por importante) pero desde la misma filosofía (Véase Garmendia, S. «El Tractatus: la paradoja salvaje». Análisis. Revista de investigación filosófica, vol. 8, n. ${ }^{\circ}$ 1, 2021, pp. 95-109). Sobre esta lectura metafilosófica del Tractatus, véase también MARTORELL, F. y SAnféLIX, V. «Pirronismo lógico. La metafilosofía del joven Wittgenstein», en Pérez Chico, D. (coord.), Wittgenstein y el escepticismo: certeza, paradoja y locura. Zaragoza: PUZ, 2019, pp. 235259, en el que los autores defienden el punto de vista según el cual Wittgenstein fue "un escéptico a propósito de la misma filosofía». Wittgenstein entiende, acertadamente desde mi modesto punto de vista, que no hay otra manera de hacerlo.

${ }_{10}$ Tal y como ya he tenido la ocasión de indicarlo, la apropiación del Tractatus por parte de la tradición analítica obedece principalmente a que en su elaboración es crucial el ascendiente que sobre el joven Wittgenstein tienen los trabajos pioneros en lógica de Frege y de Russell, pero sobre todo se debe a la recepción que del libro hacen los integrantes del Círculo de Viena. Una recepción ciertamente interesada que, si bien es en ocasiones fiel a la letra, en general traiciona el verdadero espíritu del Tractatus.

${ }^{11}$ Un gesto este, por cierto, que muestra (sin decirlo, podría añadir ahora para marcar la diferencia entre lo que sí llega a decir en el prólogo y lo que en el cuerpo del libro se muestra, sin más, confiando en que con eso basta: quiero ver en esto la evidencia clara de que Wittgenstein pone en pie de igualdad a decir y a mostrar) bien a las claras que el Tractatus no es un libro de texto (de lo contrario habría dejado la escalera para que otros subieran por ella atraídos por las enseñanzas de Wittgenstein, lo cual les ahorraría de esa manera el esfuerzo de comprender por sí mismos el libro); y también que Wittgenstein confiaba en que otros también serían capaces de llegar hasta allí arriba (comprendiendo las proposiciones del Tractatus). 
Recapitulemos: gran parte de la dificultad asociada con la posibilidad de ofrecer una interpretación cabal del Tractatus obedece a la aparente inconsistencia entre la verdad de los pensamientos contenidos en el Tractatus y que, en última instancia, estos realmente no digan nada sobre el mundo, tal y como queda reflejado en la proposición 6.54 .

Se trata de una proposición sobre cuya interpretación se ha erigido toda una industria editorial, y es una de las que más han dado que hablar a los comentaristas del Tractatus y, si avanzamos en nuestra lectura del prólogo, es fácil ver por qué.

Si este trabajo tiene algún valor, lo tiene en un doble sentido. Primero, por venir expresados en él pensamientos, y este valor será tanto más grande cuanto mejor expresados estén dichos pensamientos [...]. En este punto soy consciente de haber quedado muy por debajo de lo posible...

La verdad de los pensamientos aquí comunicados me parece, en cambio, intocable y definitiva. Soy, pues, de la opinión de haber solucionado definitivamente, en lo esencial, los problemas. Y, si no me equivoco en ello, el valor de este trabajo se cifra, en segundo lugar, en haber mostrado cuán poco se ha hecho con haber resuelto estos problemas ${ }^{12}$.

Nos encontremos entonces con que (1) la verdad de los pensamientos expresados en el libro es «intocable y definitiva»; pero (2) no dicen nada. De manera deliberadamente esquemática, sería algo así: en el Tractatus, si una proposición tiene valor de verdad, entonces tiene sentido, por lo tanto, si carece de sentido o es un sinsentido, entonces no tiene valor de verdad. Este parece ser el caso de las proposiciones del Tractatus, según afirma el propio Wittgenstein. ¿Cómo debemos interpretar esta paradoja tan evidente?, ¿qué clase de verdad es la de los pensamientos expresados por Wittgenstein si el precio que hay que pagar por expresarlos es el sinsentido?, ¿qué se propone exactamente Wittgenstein al describir las proposiciones del Tractatus, sus propios pensamientos, como sinsentidos y, por lo tanto, como algo de lo que podemos desprendernos?

La paradoja es tan obvia que Wittgenstein debe haber tenido razones poderosas para mantenerla y, además, en un lugar tan visible. Lo primero que se me ocurre pensar es que, a ojos de Wittgenstein, tanta obviedad tiene el propósito de provocar en nosotros algún tipo de reacción que nos haga pensar que no se trata de una paradoja, o que tan solo lo sea bajo ciertos supuestos que estaríamos refutando por el hecho de admitir que la función de las proposiciones puede ser otra distinta a la de comunicar verdades.

Amparados por la suposición prácticamente indiscutida de que el Tractatus no solo forma parte de la tradición analítica en filosofía en el siglo xx, sino que es uno de sus hitos fundamentales y fundantes, lo habitual a la hora de interpretarlo

12 Wittgenstein, L. Tractatus.., op. cit., p. 13. 
es suponer que la obra comunica verdades, por lo que la clave del enigma en torno a la paradoja reside en cuáles pueden ser esas verdades y en qué sentido pueden ser compatibles con el hecho de ser sinsentidos. Algo similar a esto es lo que defienden Morris y Dodd.

Al exponer su teoría del sentido, el [Tractatus] establece un límite a la expresión del pensamiento que cualquier intento de elaborar esta misma teoría del sentido. Más concretamente, si la teoría del sentido que elabora -la llamada «teoría de la figura»- es correcta, entonces intentar decir cómo deben ser el mundo y el lenguaje para que el significado sea posible es intentar decir algo sobre la forma lógica que las oraciones comparten con la realidad (TLP: 2.16-2.18); pero, según esa misma teoría, el intento de hacer tal cosa sólo puede dar lugar a un sinsentido, ya que la forma lógica no puede ser representada (TLP: 4.12). En consecuencia, si se mantiene la verdad de todo lo que ha pasado antes en el texto, entonces, al final del texto, uno se ve obligado a decir, con Wittgenstein, que lo anterior es un sinsentido (TLP: 6.54) $)^{13}$.

Y como acabamos de ver hace un momento, lo anterior nos lleva irremediablemente a una paradoja, a saber: si las proposiciones que componen el Tractatus son verdaderas, entonces son sinsentidos. Morris y Dodd defienden que es posible evitar el aire paradójico del Tractatus si negamos la teoría que nos ha conducido hasta esta aparente paradoja, es decir, la teoría figurativa del significado. Esta teoría es el eje en torno al que giran todas las proposiciones del Tractatus, ya sean las que se necesitan para fundamentarla, o las que son una consecuencia o corolario de ella. Este último parece ser el caso de la proposición 6.54, y esa es la razón por la que puede parecer que no queda más remedio que aceptar que las proposiciones del Tractatus son sinsentidos y que este es paradójico y, por lo tanto, también un sinsentido.

A Morris y Dodd les parece que es obvio que cualquier intento de defender que el Tractatus comunica verdades de algún tipo no puede escapar de la conclusión de que el Tractatus es simple y llanamente incoherente y, por lo tanto, no puede conducirnos a ninguna conclusión estable. Por su parte, Morris y Dodd optan por la alternativa obvia a la luz de lo anterior: la afirmación de que el Tractatus no comunica verdades; el Tractatus no proporciona "conocimiento proposicional, sino que su objetivo consiste en familiarizarnos con los límites del mundo: nos hace sentir esos límites (Tractatus 6.45)» ${ }^{14}$. Preguntémonos, entonces, cuál puede ser el objetivo no-alético del Tractatus y cómo se supone que Wittgenstein logra sacarlo adelante.

En este punto es fundamental que nos tomemos la importancia de la distinción entre decir y mostrar con la misma seriedad con la que se la toma Wittgenstein. Esto es lo que le confesaba a este respecto el joven Wittgenstein a Russell cuando la relación entre ellos aún era cordial:

13 Morris, M. y Dodd, J. «Mysticism and Nonsense in the Tractatus», European Journal of Philosophy, 2007, pp. 1-30.

${ }_{14}$ Ibidem, p. 5. 
El objetivo principal es la teoría de lo que puede ser expresado por las proposiciones - es decir, por el lenguaje- (y, lo que viene a ser lo mismo, lo que puede ser pensado) y lo que no puede ser expresado por proposiciones, sino tan sólo mostrado: lo cual, creo, es el problema cardinal de la filosofía ${ }^{15}$.

Si leemos este fragmento a continuación del fragmento de la carta a Von Fricker citado más arriba, no parece nada descabellado afirmar que lo que según Wittgenstein constituiría la segunda parte del Tractatus, la que no llegó a escribir, pero que aun así sería la parte importante, es tal «que no puede ser expresad[a] por proposiciones, sino tan sólo mostrad[a]». Lo dicho por Wittgenstein en 6.54, entonces, debe hacernos ver que las proposiciones del Tractatus no nos invitan a aprehender un conjunto de verdades dispuestas ante nosotros abiertamente (no las dicen), sino que, al mostrarnos lo que no puede ser dicho, nos ayudan a ver «el mundo correctamente»; y quisiera subrayar que proponer que unas proposiciones aunque no digan nada si son capaces de mostrar algo no es un premio de consolación, sino que es poner en valor una operación epistémica que está más allá del conocimiento proposicional; es otorgarle crédito o estatus epistémico a la capacidad de mostrar, y carta de naturaleza a lo que puede ser mostrado. Así, una lectura que sea caritativa con la aparente incoherencia del Tractatus no puede ser la de que lo que busca Wittgenstein es otra posición filosófica distinta acerca de la naturaleza de la representación. Lo que busca el texto es ayudarnos a adoptar otra perspectiva completamente diferente -Morris y Dodd la llaman la "perspectiva mística»- del mundo y de nuestra vida. Este, y no el de comunicar verdades, sería el verdadero propósito del Tractatus.

El Tractatus, de acuerdo con la interpretación que hacen de su objetivo Morris y Dodd, es una enmienda a cierta concepción tradicional de la filosofía, aquella cuya tarea consiste en «establecer lo que Kant denominaba verdades sintéticas a priori» ${ }^{16}$, verdades acerca de cómo debe, puede y no puede ser el mundo, lo cual, «de acuerdo con la metafísica del Tractatus [...] serían verdades acerca de la forma del mundo; y como la forma del mundo no es otra cosa que la forma general de la proposición, no puede haber, de acuerdo con el Tractatus, tales verdades ${ }^{17}$. En consecuencia, el objetivo del Tractatus, al hacernos adoptar la perspectiva mística, sería, según Morris y Dodd, hacernos ver algo que escapa a la filosofía, porque la filosofía, según ellos, pretende decir cosas, comunicar verdades. El silencio del Tractatus sería, entonces, una manera de hacer manifiesto lo que no puede ser dicho.

Morris y Dodd aceptan sin aparente conflicto que, o bien aspiramos a obtener y comunicar conocimiento proposicional y proposiciones con sentido, o bien nos conformamos con una cierta experiencia o sentimiento carente de sentido (lo místico). Según ellos, lo místico nos proporciona un conocimiento del mundo por familiaridad, pero esta caracterización en el contexto tractariano es conflictiva,

15 Carta a Russell de 1919, incluida en Wittgenstein, L. Cartas a Russell, Keynes y Moore. Madrid: Taurus, 1979, p. 124; la cursiva es mía.

${ }_{16}$ Morris y Dodd, op. cit., p. 17.

17 Ibidem. 
pues el mundo ni siquiera es una cosa, sino que es la totalidad de los hechos (Tractatus 1.1). Morris y Dodd atribuyen esta dificultad a la dificultad propia del texto y siguen adelante sin mirar atrás.

La principal virtud de la interpretación ofrecida por Morris y Dodd es que es compatible con aceptar que el Tractatus es incoherente, pues la incoherencia apunta a que su objetivo no es comunicar conocimiento proposicional ni, en consecuencia, verdades del tipo que sean (mucho menos, inefables). El objetivo del Tractatus consistiría en familiarizarnos con los límites del mundo. Según esta interpretación, el Tractatus nos lleva hasta un punto que se alcanza tan solo con posterioridad a que el texto haya sido reconocido como incoherente.

A mi modo de ver, Morris y Dodd se conforman con menos de lo que ofrece su interpretación del Tractatus. Podemos estar de acuerdo con ellos en que el Tractatus no transmite ni comunica conocimiento proposicional alguno (en realidad me siento más cómodo diciendo que no es ese su objetivo principal), pero creo que yerran al dar por hecho que esto presupone un modelo estrictamente proposicional de la comunicación, el conocimiento, la verdad y otros cognados. Es decir, no dan el que a mi modesto modo de ver es el paso necesario para poder defender que una posible enseñanza del Tractatus, condensada en la proposición 6.54, es precisamente que hay, parafraseando a Austin en "Otras mentes», una clase de conocimiento que está un paso más allá del conocimiento proposicional. Propongo avanzar en esta dirección centrando nuestra atención en el sentido exacto en el que las proposiciones del Tractatus son, como dice Wittgenstein, «elucidaciones».

El propio Wittgenstein nos echa una mano en este punto con lo que escribe en una carta enviada a Odgen al respecto de la traducción del Tractatus. En ella le dice que no hay que entender «elucidaciones» intransitivamente, sino transitivamente, esto es, que las proposiciones del Tractatus «elucidan lo que quiera que sea que elucidan de esa manera» y que piensa que esto es equivalente a decir «mis proposiciones clarifican de esta manera [...] queriendo decir con ello que 'sus proposiciones clarifican lo que quiera que sea que clarifican -por ejemplo, las proposiciones de la ciencia natural- de esta manera:...”. Y añade: «Podrías haberlo puesto de esta manera: 'mis proposiciones elucidan cuestiones filosóficas de esta manera:...' ${ }^{18}$. Es decir, no se trata de que el reconocimiento de que son sinsentidos sirva para elucidar las proposiciones del Tractatus. Antes al contrario, el reconocimiento de que se trata de sinsentidos nos dice que las proposiciones ayudan a elucidar otra cosa, algo distinto de ellas. En otras palabras: estas proposiciones son esencialmente sinsentidos, su tarea o propósito no es decir verdades, por lo tanto, decir que son sinsentidos no debe ser visto como un demérito.

$\mathrm{Al}$ no tomarse lo suficientemente en serio la seriedad con la que Wittgenstein propone la distinción entre decir y mostrar, a Morris y Dodd se les escapa la

18 Wittgenstein, L. Letters to C.K. Odgen with Comments on the English Translation of the Tractatus Logico-Philosophicus. Von Wright, G.H. (ed.). Londres: Routledge \& Kegan Paul, 1973, p. 51 
posibilidad de reunir en una alternativa a las expuestas en su trabajo la idea de que, aunque es posible que el Tractatus no comunique verdades proposicionales, no es incompatible con que se puede comunicar una verdad mostrándola y no solo diciéndola. Esto, aun siendo aparentemente compatible con la opción que defiende que el Tractatus comunica exclusivamente verdades inefables (mostrándolas), va un poco más lejos que ella porque no caracteriza la operación de mostrar como si fuera un premio de consolación cuando no es posible decir nada ${ }^{19}$. Y también porque, al tomarse tan en serio como corresponde la potencia epistémica de mostrar, otorga carta de naturaleza a una región que trasciende y envuelve al mundo y a la capacidad de representarlo. Y una vez localizada esta región no se trata de que podamos encontrar la manera de poner en palabras lo importante, sino de reivindicar otro tipo de pensamiento diferente al que está limitado por el formalismo de la teoría figurativa, uno que no se deja nada fuera.

III

Es de esperar que la afirmación de Wittgenstein en el prólogo de que su libro comunica algunas verdades que le parecen incuestionables se interprete como si esas verdades, y el conocimiento de las mismas, tuvieran que ser necesariamente proposicionales. Resulta también natural pensar que el Tractatus ofrece una respuesta realista a la siguiente pregunta: ¿¿de qué manera logra una oración tener sentido y que así un hablante pueda comunicársela a un oyente? ${ }^{20}$, ¿qué otra cosa, si descartamos el mundo, puede proveer de sentido a nuestras oraciones? Todo parece hablar a favor de esta opción y ni siquiera nos imaginamos de qué otra manera podría ser o, si logramos imaginárnosla, lo normal es que consideremos que se trata de una obviedad $^{21} \mathrm{o}$ algo peor.

Sin salir del prólogo del Tractatus, al final del mismo, justo después de haber descrito la verdad de los pensamientos de los que se da cuenta en el Tractatus como «intocable y definitiva», Wittgenstein presume de haber «resuelto los problemas de modo indiscutible», pero inmediatamente elimina cualquier conato de presunción por su parte por medio de la siguiente declaración: «la segunda cosa de valor que hay

19 Así, por ejemplo, podemos ser perfectamente conocedores de todo lo que se puede decir de alguien (todas sus verdades proposicionales, por así decirlo) y sin embargo no conocer a esa persona en absoluto. Debo este ejemplo a Vicente Sanfélix.

${ }^{20}$ Véase Pears, D. Paradox and Platitude in Wittgenstein's Philosophy. Oxford: Clarendon Press, 2006, p. 1.

${ }^{21}$ De nuevo Pears ofrece esta explicación como respuesta a la persistencia y resiliencia de las teorías realistas en filosofía a pesar de sus limitaciones manifiestas. Es un tipo de explicaciones que no identifica la causa con independencia del efecto: «I call this flower blue because I recognize that that is its color [...] but my statement owed its sense to the previous correlation of the world with the colour, and it is not so easy to see how that correlation should be characterized» (ibidem, p. 2). Usamos la palabra «azul» para referirnos al color azul porque la palabra «azul» está correlacionada con el color azul. Y esto no explica nada, no es informativo, es una petición de principio. 
en este trabajo consiste en mostrar cuán poco se ha conseguido una vez que estos problemas se han resuelto». Y en el cuerpo principal del Tractatus añade lo siguiente: «Sentimos que, aún cuando todas las posibles preguntas científicas hayan obtenido una respuesta, nuestros problemas vitales ni siquiera se han tocado» (Tractatus 6.52). Parece que después de que se ha dicho todo lo que es posible decir, de que se ha contestado a todas las preguntas que es posible formular, es muy poco lo que se ha dicho. $\mathrm{O}$ al menos sentimos que hay algo más allá de los límites de nuestro conocimiento del mundo, aunque sobre ello no podamos decir nada. ¿Qué importancia puede tener que no tenga cabida en la imagen científica del mundo?

Sabemos que, según el Tractatus, una proposición tiene sentido solo si es una figura de la realidad. Esto expulsa de dicho ámbito tanto a las proposiciones de la lógica como a los sinsentidos. A las primeras porque ellas son las condiciones de posibilidad del sentido (de hecho, lo correcto sería llamar «espacio lógico» a lo que he llamado «ámbito de sentido»), de tal manera que se sitúan en el mismo límite del sentido - en realidad lo constituyen-; a las segundas porque no son figuras de la realidad. A modo de corolario diré que esta expulsión del paraíso del sentido tiene otra consecuencia: las proposiciones de la lógica y los sinsentidos también son impermeables a la verdad. Efectivamente, tan solo una figura de la realidad, una proposición con sentido, por ejemplo, posee valor de verdad, porque solo una proposición que diga algo sobre el mundo (solo una proposición que represente o describa un estado de cosas) puede ser juzgada en términos de verdad o falsedad. ¿Qué proposiciones son las que tienen por objetivo describir el mundo? Las proposiciones de las ciencias naturales: «la totalidad de las proposiciones verdaderas es la ciencia natural al completo (o la totalidad de las ciencias naturales)» (Tractatus 4.11). En el Tractatus, Wittgenstein se preocupa de dejar bien claro que ni la lógica ni la filosofía pertenecen a las ciencias naturales:

La filosofía no es ninguna de las ciencias naturales.

(La palabra filosofía tiene que significar algo que esté por encima o por debajo de las ciencias naturales, pero no a su mismo nivel) (Tractatus 4.111).

$\mathrm{Al}$ sentido de una proposición le corresponde un lugar en el espacio lógico. Es algo que puede ser o no ser el caso (cf. Tractatus 4.1). Es contingente. La verdad, entonces, es una propiedad de lo contingente: depende de cómo es, de hecho, el mundo. Pero cómo es el mundo no es lo importante: «Para lo que es más elevado resulta absolutamente indiferente cómo sea el mundo» (Tractatus 6.432); «[l]o místico [i.e.: lo inefable, ergo, lo importante según reconoce Wittgenstein en su carta a Von Fricker] no consiste en cómo es el mundo, sino en que sea» (Tractatus 6.44).

Cuando en el Tractatus Wittgenstein se pregunta por los límites del sentido, lo normal es pensar que lo que trata de averiguar es cómo adquieren sentido las oraciones, esto es, cómo pueden ser comunicadas y comprendidas. Pero la respuesta, como sabemos, ya está predeterminada: el sentido al que se refiere Wittgenstein es un sentido fáctico, depende única y exclusivamente de cómo es (o puede ser) el mundo. Según la teoría figurativa, tener sentido es, en definitiva, ser comunicable. Lo que se puede comunicar, entonces, se limita a lo que tiene sentido fáctico. 
Se sigue de lo anterior que si lo que puede ser dicho es una prerrogativa de las disciplinas descriptivas que figuran el mundo, entonces estas no tienen nada que decir sobre lo importante. El ámbito de la ciencia es, como acabamos de ver, el ámbito de lo contingente, de lo que podría haber sido de otra manera. La importancia de lo importante, lo inefable, entonces, reside en que su reconocimiento nos hace sentir que una imagen científica y reduccionista de la realidad no tiene lugar para lo importante. Sea cual sea la función que puedan tener las proposiciones que nos ayudan a reconocer lo inefable, lo que es seguro es que no es representacional y/o descriptiva, sino que su función debe servir más bien para provocar un cambio de actitud a propósito de la vida y del mundo en quienes las lean comprendiéndolas. Nos ayudan a adoptar la perspectiva mística y esta es una perspectiva ética, es la perspectiva correcta del mundo ${ }^{22}$.

Volvamos brevemente a las preguntas suscitadas por 6.54, concretamente a la tercera, y dentro de esta, al final: en tanto sinsentidos no solo podemos, sino que debemos, desprendernos de las proposiciones del Tractatus. Ahora bien, ¿cuándo debemos desprendernos de ellas?, ¿nos sume este gesto es una actitud quietista? Lo que parece insinuar 6.54 es que debemos sacar partido de estas proposiciones para, usándolas como si fueran los peldaños de una escalera, llegar a una posición elevada desde la que sea posible ver «el mundo correctamente».

La visión objetiva del mundo, como acabamos de ver, no es la visión correcta porque es a todas luces incompleta (esta es una conclusión a la que nos conduce el rigor lógico y la coherencia interna del Tractatus). Además de los hechos que constituyen el mundo -lo contingente-, está lo místico -lo absolutamente incondicionado-. Y está también nuestra valoración de estos. Afirmar, como hace Wittgenstein, que el mundo del hombre feliz es distinto del mundo del hombre infeliz (Tractatus 6.43), ciertamente no nos dice nada acerca de cómo es, de facto, el mundo, pero sí modula nuestras representaciones del mismo. Decir cómo es tal o cual estado de cosas es, qué duda cabe, importante, pero no es lo más importante si existen diferentes maneras de valorar nuestras representaciones del mundo. El valor es lo que dota de interés al mundo, pero el valor reside fuera del mundo (Tractatus 6.41), pues el mundo está constituido únicamente por hechos y por lo tanto el valor no forma parte de él.

La disciplina que se ocupa del valor es la ética. Wittgenstein nos dice que la ética, como la lógica, es una condición del mundo ${ }^{23}$. No trata sobre el mundo, sino que lo trasciende. Ahora bien, ¿̇e qué es condición la ética? A la ética le concierne la posibilidad de un modo particular de experimentar el mundo: experimentarlo como algo valioso o significativo.

22 Cf. Morris y Dodd, op. cit., p. 15.

23 Wittgenstein, L. Cuadernos de notas (1914-1916). Madrid: Síntesis, 2009, p. 215. 
Queda claro, espero, que para el autor del Tractatus la ética no es sobre los estados de cosas que conforman el mundo, y que su objeto -por ejemplo, el valor, o el sentido de la vida, o qué es lo que hace que merezca la pena vivir la vida, o cuál es la manera correcta de vivir ${ }^{24}$ - es inefable, esto es, no es figurable, por lo tanto, no puede haber, estrictamente hablando, proposiciones éticas: la ética no se puede enunciar ${ }^{25}$. No hay una ciencia ética. Y si, como confiesa Wittgenstein a Von Fricker, el propósito del Tractatus es ético, entonces, esa es otra razón por la que el Tractatus al completo es un sinsentido, pero no por error o descuido, sino que lo es esencialmente: su propósito no es decir cómo es $e l$ mundo, ni mucho menos cambiarlo, sino, en primer lugar, poner en valor lo que cae fuera del mismo. Este es, pues, el sentido ético del Tractatus: la visión correcta que logramos si seguimos el camino pavimentado por sus proposiciones no es una que sirva para conocer con certeza cómo son las cosas, sino que separa aquello que puede ser conocido de lo que no puede serlo. Con este reconocimiento nos posicionamos en el mundo de una determinada manera (cf. Tractatus, 4.115).

¿Cómo entran en contacto, por así decir, la ética y el mundo si, como afirma Wittgenstein, el punto de vista de la ética (y de la estética) es el punto de vista sub especie aeterni? La respuesta es: por medio del sujeto. «El mundo de la representación", nos dice Wittgenstein, «no es ni bueno ni malo, sino que lo es el sujeto que quiere ${ }^{26}$. "Bien y mal sólo entran en escena a través del sujeto», que es un límite del mundo ${ }^{27}$. Pero no queda aquí la cosa, sino que «el mundo es mi mundo" (Tractatus 5.641; el subrayado es mío). Esto es, para mí (y para cualquiera), el mundo es el mundo de los objetos que puedo nombrar, de ahí también que «[l]os límites de mi lenguaje significan los límites de mi mundo" (Tractatus 5.6): solo puedo nombrar aquello cuya existencia conozco. Esos son los objetos que componen los estados de cosas que soy capaz de representar y los nombres cuya concatenación dará lugar a las proposiciones que para mí tienen sentido, el conjunto de las cuales constituye mi lenguaje (el único que entiendo [Tractatus 5.62], esto es, el conjunto de todas las proposiciones que para mí tienen sentido ${ }^{28}$. Así que, aunque una consecuencia de la teoría figurativa es que el solipsismo es correcto (Tractatus 5.62), la verdad del solipsismo no es que tan solo existo yo y mis pensamientos, sino que mi mundo está constituido por los hechos que para mí tienen sentido. Pero mi mundo no es un objeto que experimento como si fuera algo ajeno, sino que es el mundo que vivo y no solo el mundo en el que vivo (cf. Tractatus 5.621). Así que está en mi mano que mi mundo sea el de un hombre feliz, pongamos por caso.

${ }^{24}$ Cf. Wittgenstein, L. Conferencia sobre ética. Barcelona: Paidós, 1989, p. 5.

25 Wittgenstein, L. Cuadernos de notas..., op. cit., p. 217.

${ }^{26}$ Ibidem, p. 219.

${ }^{27}$ Ibidem. Cf. Tractatus 5.632.

28 SAnfélix, V. «Tractatus 5.6-5.621», Teorema XL/2, 2021, p. 141. 

dogmático?

¿Nos despierta la paradoja del Tractatus de un sueño representacionalista

La teoría figurativa conduce a paradojas, como por ejemplo a que el solipsismo sea verdadero o, en general, que las proposiciones del Tractatus expresen pensamientos cuya verdad es incontestable a pesar de que sean sinsentidos. He tratado de sortear esta amenaza de incoherencia mostrando que está al servicio de algo mayor que escapa los límites de la lógica, aunque es gracias a la lógica como llegamos a ser conscientes de ella. El Tractatus constituye un esfuerzo prometeico por parte de Wittgenstein para hacer inteligible lo místico, contra la concepción realista/representacionalista del sentido que convierte en algo inefable y por lo tanto inútil para lidiar con nuestros problemas vitales, quedando, así, desatendidos.

Wittgenstein nos avisa desde el principio que el libro pretende establecer los límites del sentido. Dichos límites son tales que más allá de ellos no puede haber nada que pueda ser pensado. Si lo hubiera sería ilógico, y lo ilógico no tiene cabida en el espacio lógico de posibilidad. Pero lo que esto quiere decir exactamente es que más allá del límite de sentido no hay nada que pueda ser caracterizado en términos de verdadero o falso porque es lo absolutamente incondicionado. El límite, entonces, lo es también para la clase de inteligibilidad que puede proporcionar la ciencia natural acerca de nuestro mundo.

Mi relación con el mundo es algo que experimento en silencio. El silencio sirve para situar cada cosa en su sitio: lo importante y lo que no lo es tanto; lo místico y lo fáctico; el sinsentido y el sentido; el valor y la verdad; lo absolutamente incondicionado y lo contingente.

Se me ocurren pocos silencios tan elocuentes como el silencio en el que se sume el Tractatus al final: es una muestra de reconocimiento y al mismo tiempo una invitación para que otros alcancen por sus propios medios la visión correcta (por ejemplo, ser conscientes de lo que tenemos por importante). Lo real no sería solo lo que puede ser descrito a partir del, o gracias al, isomorfismo entre mundo y lenguaje. Lo real es lo irremplazable. Aquello de lo que no podemos privarnos sin reducir drásticamente nuestro mundo. El silencio del Tractatus, si acaso, se opone al ruido y a la cháchara inconsecuente, pero no al intento por comprender con palabras. El Wittgenstein maduro convertirá esta naturaleza lingüística de nuestra forma de vida en el santo y seña de su filosofía, pero hay ya en el Tractatus un primer atisbo de que el tipo de conocimiento que obtenemos a través de la correspondencia de nuestras representaciones del mundo no es suficiente.

Me gustaría haber sido capaz de mostrar que el Tractatus es, sobre todo, una puerta de entrada al ámbito de lo inefable. Si juntamos en una misma frase que (1) el libro transmite algunas verdades; (2) que el propósito del libro es ético; (3) que de acuerdo con la teoría figurativa las proposiciones éticas carecen de sentido; (4) que el sentido del que se habla en el Tractatus es fáctico; (5) que al trazar los límites del sentido se ha logrado bien poco; y (6) que lo importante es lo que ha quedado más allá de los límites del sentido, creo que es posible concluir que lo que persigue Wittgenstein con su argumentación aparentemente incoherente es mostrar las limitacio- 
nes de una concepción realista/representacionalista de la relación del pensamiento y el lenguaje con el mundo, como por ejemplo que no caben en ella algunas verdades sobre mi mundo y mi vida (sobre el mundo y la vida de cualquiera de nosotros).

Que Wittgenstein no especifique en qué consiste exactamente la manera de lidiar con el mundo que no es representacionalista ni proposicional tampoco debe ser juzgado con severidad porque su propósito no es (y ha mostrado que no puede ser) pontificar desde lo alto de la atalaya a la que le han conducido sus pensamientos. Y, al fin y al cabo, tampoco fue capaz de poner ejemplos de qué son exactamente los objetos que nombran los nombres y ningún intérprete se rasga las vestiduras por ello. ¿Nos fuerza la conclusión del Tractatus a habitar nuestro mundo en silencio? Las decisiones vitales del propio Wittgenstein tras su regreso a Viena al finalizar su participación en la Gran Guerra así parecen sugerirlo. Pero se trata de un silencio que es compatible con que el mensaje final sería que la verdad de lo importante es algo que hay que percibir, sentir o reconocer por uno mismo, pero está al alcance de todos. Si esto es así, entonces la proposición 6.54 es en realidad la última proposición del Tractatus, y el silencio forma parte ya de la visión correcta. 
